\title{
AUTOMATIC 3D BUILDING MODEL GENERATIONS WITH AIRBORNE LiDAR DATA
}

\author{
N. Yastikli a, *, Z. Cetin ${ }^{\text {a }}$ \\ ${ }^{a} Y T U$, Civil Engineering Faculty, Department of Geomatics Engineering, 34210 Davutpasa, Istanbul, Turkey - \\ (ynaci, zerisir)@yildiz.edu.tr
}

KEY WORDS: 3D modelling, LiDAR, Point Cloud, Classification, Point Based Classification

\begin{abstract}
:
LiDAR systems become more and more popular because of the potential use for obtaining the point clouds of vegetation and manmade objects on the earth surface in an accurate and quick way. Nowadays, these airborne systems have been frequently used in wide range of applications such as DEM/DSM generation, topographic mapping, object extraction, vegetation mapping, 3 dimensional (3D) modelling and simulation, change detection, engineering works, revision of maps, coastal management and bathymetry. The 3D building model generation is the one of the most prominent applications of LiDAR system, which has the major importance for urban planning, illegal construction monitoring, 3D city modelling, environmental simulation, tourism, security, telecommunication and mobile navigation etc. The manual or semi-automatic 3D building model generation is costly and very time-consuming process for these applications. Thus, an approach for automatic 3D building model generation is needed in a simple and quick way for many studies which includes building modelling. In this study, automatic 3D building models generation is aimed with airborne LiDAR data. An approach is proposed for automatic 3D building models generation including the automatic point based classification of raw LiDAR point cloud. The proposed point based classification includes the hierarchical rules, for the automatic production of 3D building models. The detailed analyses for the parameters which used in hierarchical rules have been performed to improve classification results using different test areas identified in the study area. The proposed approach have been tested in the study area which has partly open areas, forest areas and many types of the buildings, in Zekeriyakoy, Istanbul using the TerraScan module of TerraSolid. The 3D building model was generated automatically using the results of the automatic point based classification. The obtained results of this research on study area verified that automatic 3D building models can be generated successfully using raw LiDAR point cloud data.
\end{abstract}

\section{INTRODUCTION}

Airborne laser scanning (ALS) is one of the fast and accurate active remote sensing techniques to map Earth topography, and obtained results are 3D point clouds (Mallet, 2010). The reduction of the system cost and the improvement of the LiDAR sensors accuracy enable the high level automation in data collecting, as a result, LiDAR point cloud data have been used in many fields (Forlani et al., 2006; Gu et al., 2016). The 3D city modelling, Digital Terrain Model (DTM) generation, bridge and power line detection, corridor, coastal or opencast mapping, forest management, change detection are the some of the application which uses the LiDAR data as well as metrology and cultural heritage (Sithole, 2005; Mallet, 2010).

3D city model is a digital representation of the Earth's surface and its related objects such as building, tree, vegetation, and some man-made feature which belong to urban area (Singh et al., 2013). In the last decades, 3D city models generally have been utilised for visualisation. However, nowadays 3D city models are increasingly employed in a number of different domains and for a large range of tasks beyond visualisation, e.g. estimation of solar irradiation, energy demand estimation, aiding positioning, determination of floor space and classifying building types (Biljecki et al., 2015; Liang et al., 2016). The generated buildings in this $3 \mathrm{D}$ city models also have many useful applications such as urban planning, cartography, surveillance, wireless network planning, entertainment, virtual tourism, geo-spatial web browsing, etc. (Verma et al., 2006). However, producing 3D building models for a variety of applications is very time consuming and challenging task by manual or semi-automatic methods. Nowadays, generation of 3D building models in a simple and quick way has become important. A typical 3D building model is derived from various acquisition techniques, for instance, photogrammetry and laser scanning, synthetic aperture radar, architectural models and drawings (Biljecki vd., 2015). LiDAR point cloud data improves the success of the automated 3D building model generation methods (Oude Elberink and Vosselman, 2009; Uzar Dinlemek, 2012). LiDAR data processing steps are also very prominent for 3D building models production applications and should be handled in proper ways.

The LiDAR point classification is the first step of LiDAR data processing in many applications such as 3D city modelling, building extraction, DEM generation. Therefore, it is necessary to classify the LiDAR point cloud correctly in order to obtain highly accurate results in such studies (Charaniya et al., 2004). In classification steps of the LiDAR point clouds, each point is assigned to the meaningful classes such as ground, vegetation, building according to the characteristics of the LiDAR data (Yastikli and Cetin, 2016). Algorithms for classifying LiDAR point clouds can be grouped into two categories based on used data type: point clouds and raster range image. The first kind of algorithms is used on point clouds of LiDAR directly and second kind of algorithms work with a raster image, so the irregularly distributed LiDAR point cloud must be gridded (Bao et al., 2008). In second approach, most of the time LiDAR point cloud data transformed into image data, so the traditional pixelbased classification or the object-based segmentation and classification techniques can be directly used for LiDAR data

\footnotetext{
* Corresponding author
} 
classification ( $\mathrm{Gu}$ et al., 2016). However, when the LiDAR point data are resampled into a grid space, the $3 \mathrm{D}$ data are converted into $2 \mathrm{D}$ grid and this process cause data losses in details such as building edges (Bao et al., 2008; El-Ashmawy and Shaker, 2014). Compared with the pixel in image, the LiDAR point cloud data can retain more 3D spatial information, because of that it is crucial to propose a methodology which use the original (raw) LiDAR point cloud data for urban land cover classification (Gu et al., 2016). For this reason, the use of the raw LiDAR point cloud used in the classification improves the accuracy of application.

In this study, automatic generation of simple 3D building models were aimed with raw airborne LiDAR data. An approach for the production of automatic 3D building model has been proposed and tested in Zekeriyakoy, Istanbul. Considering this aim the paper is organized as follow: the next section describes the details of the automatic point based classification of raw LiDAR point cloud and automatic generation of 3D building models. This is followed by the study area, data set and the experimental section which provides the detailed information about automatic classification of the raw point cloud and 3D building models generation. The concluding remarks are given in last section.

\section{METHODOLOGY}

Classification of the LiDAR point cloud including building class is very critical for the $3 \mathrm{D}$ building models generation. In point classification, each irregularly distributed 3D LiDAR point is assigned to a semantic object class which it is belongs to (Niemeyer et al., 2014; Charaniya et al., 2004). The features of all points in the LiDAR point cloud are used in automatic point-based classification applications and appropriate parameters of these features are usually determined as a result of training steps according to the targeted classes in the classification stage (Kim and Sohn, 2010; Mallet et al., 2011; Kim and Sohn, 2013, Yastikli and Cetin, 2016). The used features in point based classification algorithms can be divided into 3 groups as spatial-based features, echo-based features and waveform-based features (Mallet et al., 2011). The local environment, height features, eigenvalue features, local plane features, surface-based features and vertical profile features are some of the popular spatial-based features (Kim and Sohn, 2010; Mallet et al., 2011; Kim and Sohn, 2013; Chehata et al., 2009). Multiple reflections of laser signal such as single return, first return, intermediate return and last return and different combinations of these returns are the echo-based features (Kim and Sohn, 2010). Echo amplitude, echo width, echo shape and echo cross-sections are waveform-based features can be obtained only full-waveform LiDAR systems (Chehata et al., 2009).

In this study, a classification approach was proposed that allow automatic point-based classification of point clouds for 3D building models generation using airborne LiDAR data. The proposed point-based classification method aims to separate the raw LiDAR point cloud data into the three basic land cover classes (ground, vegetation, and building). The features are determined for the classification and the hierarchical rule set was created for proposed approach with defined routines. The proposed approach includes both spatial and echo-based features. Figure 1 shows the workflow of the proposed strategy for $3 \mathrm{D}$ building models generation.

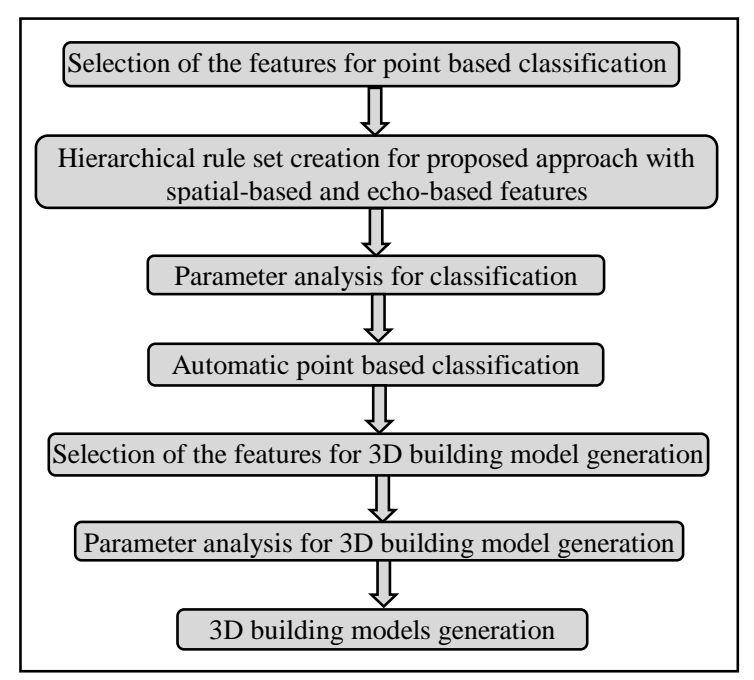

Figure 1. Workflow of the proposed 3D building models generation

In the point-based classification approach, each point is analysed according to its properties and automatically assigned to the belonging class using the created hierarchical rule sets. The most important point of this classification is deciding to the parameters which are used in the hierarchical rule sets. When the parameter values are optimum in the study area, the classification results also will be much more accurate. Therefore, detailed parameter analyses were performed to determine the parameters to be used in the rule sets for ground and building classes in order to ensure high classification accuracy. These analyses were performed in different pilot areas selected in the study area. In Figure 2, ortho image of the selected pilot area 1 , the classification results with and without parameter analyses for ground are given. Similarly, ortho image of the selected pilot area 2, the classification results with and without parameter analyses for building class, are shown in Figure 3.
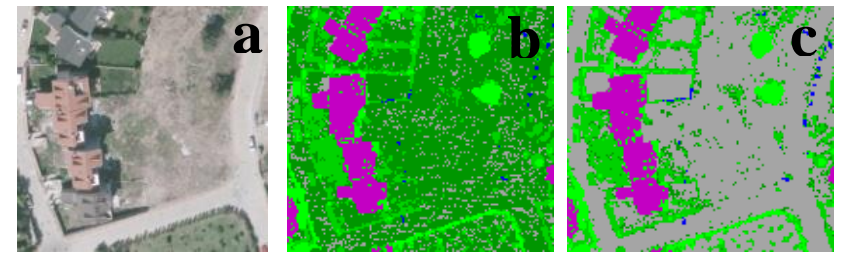

Figure 2. Ortho image of the selected pilot area 1, the classification results without (b) and with (c) parameter analyses for ground classes
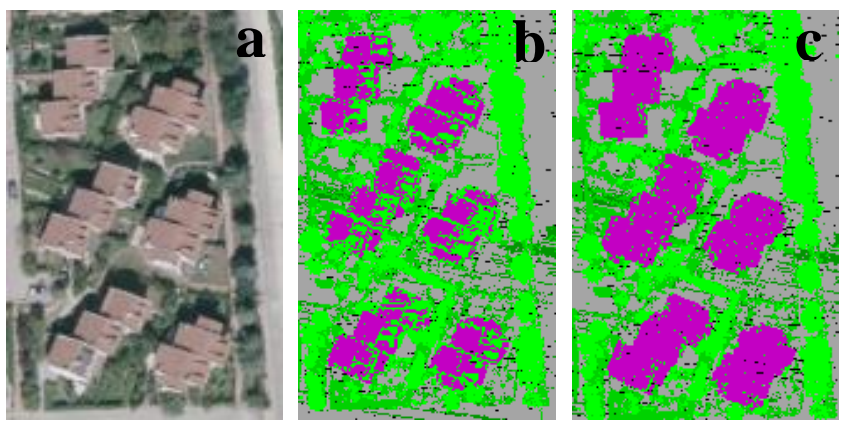
Figure 3. Ortho image of the selected pilot area 2, the classification results without (b) and with (c) parameter analyses for building classes

\section{GENERATION OF AUTOMATIC 3D BUILDING MODELS}

For the generation of simple 3D building models, Zekeriyakoy, which is located in the Saryer district of Istanbul, is determined as the study area. The study area has small open areas and forest areas and many buildings. The ortho image of the study area is given in Figure 4a. The LiDAR data of the study area was collected with 'Riegl LSM-Q680i' full-waveform laser scanner mounted 'Eurocopter AS350' in September, 2013. The obtained point cloud from Istanbul Metropolitan Municipality with the density of 16 points $/ \mathrm{m}^{2}$ was in LAS (Log ASCII Standard) format and classified with standard parameters including the ground, low vegetation, medium vegetation, high vegetation, building, low point and air point classes (see Figure $4 \mathrm{~b}$ ).
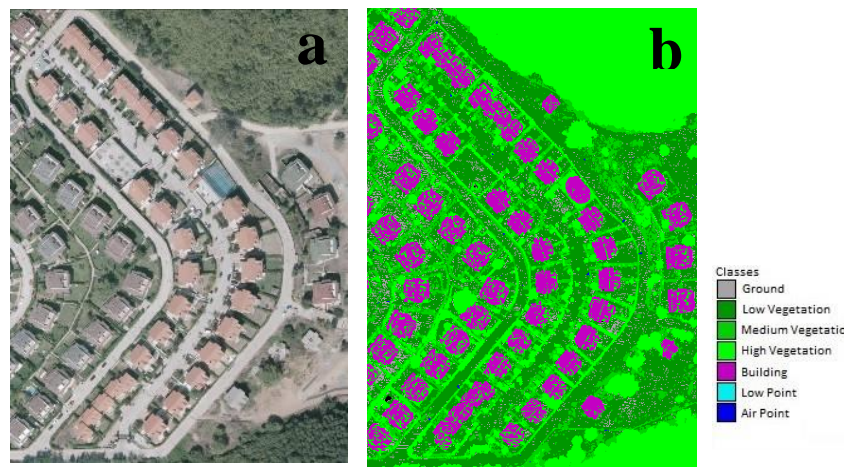

Figure 4 . The ortho image of the study area (a) and roughly classified point cloud with standard parameters (b)

The point cloud data of the study area was classified by the proposed point-based approach, which use the spatial and echobased features of LiDAR points, as a first step of the generation of automatic 3D building model. TerraScan module of TerraSolid software was used for the classification process. The proposed approach used in this classification automatically performs the classification process with the hierarchical rule sets created by using the parameters determined as the result of the parameter analysis. The obtained classes were ground, low vegetation, medium vegetation, high vegetation, building, default, low point and air point as a result of the automatic point based classification. The low, medium and high vegetation classes were combined into a single 'vegetation' class and default, low point and air point classes were also combined into a single 'other' class at the end of the automatic classification process. Figure $5 \mathrm{a}$ shows the $3 \mathrm{D}$ view of the automatic point based classification results using the spatial and echo-based features of LiDAR points for the study area. The ground class obtained more accurately with the proposed approach in comparison to the roughly classified point cloud (Figure $4 \mathrm{~b}$ ) using the standard parameters. A large majority of roads passing through buildings in the study area were correctly assigned to the ground and the building classes using the proposed approach. The obtained classification results verify the success of the proposed automatic point-based classification method for $3 \mathrm{D}$ building model generation.

After classification step, simple 3D building models were produced in the study area using the building points classified by proposed approach based on the spatial and echo-based features of LiDAR points. First, parameters were determined for the features such as planarity, slope, area, etc. based on the classified building points and then the $3 \mathrm{D}$ building models were generated automatically using these determined parameters in TerraScan module of TerraSolid. Figure $5 b$ shows the generated $3 \mathrm{D}$ building models in study area. A closer 3D view of the automatically generated $3 \mathrm{D}$ building models which bordered with red rectangle in Figure $5 \mathrm{~b}$ is given in Figure $6 \mathrm{a}$ and the $3 \mathrm{D}$ building models overlapped with building class points is given in Figure $6 \mathrm{~b}$. When the $3 \mathrm{D}$ building modelling results are examined, it is seen that the 3D models can be successfully produced for the buildings using classified building points with the proposed approach. It is possible to use proposed approach to achieve realistic $3 \mathrm{D}$ model which require building models such as 3D city model production.
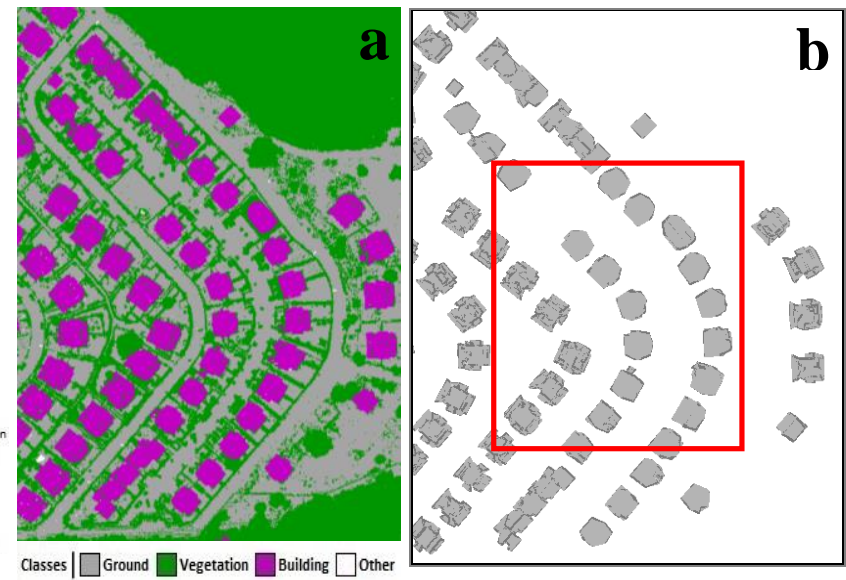

Figure 5. Automatic point based classification results of proposed approach (a) and 3D building models generated using building class points (b)
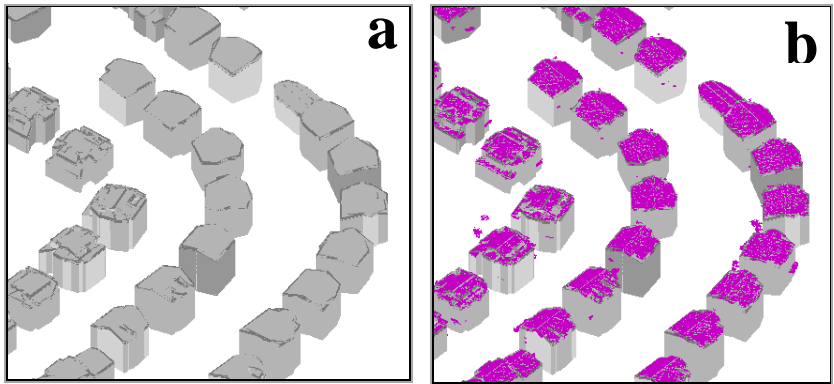

Figure 6. A closer 3D view of the automatically generated 3D building models (a) and 3D building models overlapping building class points obtained using proposed approach (b)

\section{CONCLUSIONS}

In this study, 3D building models generation was carried out with raw airborne LiDAR data to use in studies like 3D city modelling. A point based classification approach based on hierarchical rules was proposed as a result of detailed parameter analyses in pilot areas for the accurate and quick 3D building model generations. The airborne LiDAR data of the study area in Zekeriyakoy, Istanbul was classified by the proposed approach based on the spatial and echo-based features.

One of the most critical issues in this automatic 3D building model generation study is the classification of the LiDAR point 
cloud. The determination of the more realistic parameters in the rule sets for point based classification will increase the classification accuracy. Because of the fact that, the detailed parameters analyses have been performed in the pilot area 1 and pilot area 2 in study area separately. The more accurate 3D building models were generated using the improved classification results with the help of the detailed parameters analyses.

The obtained results of this research on study area verified that automatic 3D building models can be generated successfully using raw LiDAR point cloud data. The produced 3D building models can be used in different applications such as 3D city modelling, environmental simulation, telecommunication and mobile navigation. According to this study, classification of airborne LiDAR points with point-based classification method is very important for 3D building models generations correctly so that point-based classification of raw LiDAR data using hierarchical rules obtained with detailed parameter analysis is highly recommended. As the accuracy of classification increases, the accuracy of 3D building models can be increased.

\section{ACKNOWLEDGEMENTS}

We would like to thank the Metropolitan Municipality of Istanbul for the LiDAR data set of the test area.

\section{REFERENCES}

Bao, Y., Guoping, L., Chunxiang, C., Xiaowen, L., Hao, Z., Qisheng, H., Linyan, B., Chaoyi, C., 2008. Classification of LiDAR Point Cloud and Generation of DTM From LiDAR Height and Intensity Data In Forested Area, Internat. Arch. Photogram. Remote Sensing Spatial Inform. Sci., XXXVII(3/W19): 313-318.

Biljecki, F., Stoter, J., Ledoux, H., Zlatanova, S., Çöltekin, A., 2015. Applications of 3D city models: state of the art review, ISPRS Int. J. Geo-Inform. 4, 2842-2889.

Charaniya, A. P., Manduchi, R., Lodha, S. K., 2004. Supervised Parametric Classification of Aerial Lidar Data, IEEE Workshop on Real Time 3D Sensor and their Use, June 2004, Washington DC.

Chehata, N., Guo, L., Mallet, C., 2009. Airborne LiDAR Feature Selection For Urban Classification Using Random Forests, In: The International Archives of the Photogrammetry, Remote Sensing and Spatial Information Sciences, 39 (Part 3/W8): 207-212.

El-Ashmawy, N., Shaker, A., 2014. Raster vs. point cloud LiDAR data classification. Int. Arch. Photogramm. Remote Sens. Spat. Inform. Sci. 2014, 40, 79.

Forlani, G., Nardinocchi, C., Scaioni, M., Zingaretti, P., 2006. Complete classification of raw LIDAR data and 3D reconstruction of buildings, Pattern Analysis \& Applications, 8:357-374. DOI:10.1007/s10044-005-0018-2.

Gu, Y., Wang, Q., Xie, B., 2016. Multiple kernel sparse representation for airborne LiDAR data classification. IEEE Trans. Geosci. Remote Sens. 2016, 1-21.

Kim, H. B., Sohn, G., 2010. 3D Classification of Power-Line Scene From Airborne Laser Scanning Data Using Random
Forests, ISPRS Archives, September 1-3, Saint-Mandé, France, 38(3A): 126-132.

Kim, H. B., Sohn, G., 2013. Point-Based Classification of Power Line Corridor Scene Using Random Forests, Photogrammetric Engineering and Remote Sensing, 79: 82133.

Liang, J., Gong, J., Liu J., Zou, Y., Zhang, J., Sun, J., Chen, S., 2016. Generating Orthorectified Multi-Perspective 2.5D Maps to FacilitateWeb GIS-Based Visualization and Exploitation of Massive 3D City Models, ISPRS Int. J. Geo-Inf. 2016, 5, 212; doi:10.3390/ijgi5110212.

Mallet, C., 2010. Analyse des données lidar aéroportées à Retour d'Onde Complète pour la cartographie des milieux urbains. PhD Thesis. Signal and Image Processing. Télécom ParisTech, 2010. French. <pastel-00566992>

Mallet, C., Bretar, F., Roux, M., Soergel, U., Heipke, C., 2011. Relevance Assessment of Full-Waveform Lidar Data For Urban Area Classification, ISPRS Journal of Photogrammetry and Remote Sensing, 66(6): 71-S84.

Niemeyer, J., Rottensteiner, F., Soergel, U., 2014. Contextual Classi- Fication of LiDAR Data and Building Object Detection In Urban Areas, ISPRS Journal of Photogrammetry and Remote Sensing, 87: 152-165.

Oude Elberink, S., Vosselman, G., 2009. Building Reconstruction by Target Based Graph Matching on Incomplete Laser Data: Analysis and Limitations, Sensors, 9(8): 61016118.

Singh, P. S., Jain, K., Mandla, V. R., 2013. Virtual 3D city modeling: techniques and applications. In: The International Archives of the Photogrammetry, Remote Sensing and Spatial Information Sciences, ISPRS 8th 3DGeoInfo Conference, Istanbul, Volume XL-2/W2, pp. 73-91.

Sithole, G., 2005. Segmentation and Classification of Airborne Laser Scanner Data. PhD Thesis, ITC, University of Delft, The Netherlands, ISBN: 9061322928.

Uzar Dinlemek, M., 2012. Otomatik Bina Çıkarımı Uygulamalarında Çoklu Algılama Sistemi Kullanım Olanaklarının Analizi, PhD Thesis, YTU Graduate School of Natural and Applied Sciences, Istanbul.

Verma, V., Kumar, R., Hsu, S., 2006. 3D Building Detection and Modeling from Aerial LIDAR Data. Computer Vision and Pattern Recognition, IEEE Computer Society Conference, vol.2, pp. 2213-2220, doi: 10.1109/CVPR.2006.12.

Yastikli, N., Cetin, Z., 2016. Classification of LiDAR Data With Point Based Classification Methods, Int. Arch. Photogramm. Remote Sens. Spatial Inf. Sci., XLI-B3, 441-445, doi:10.5194/isprs-archives-XLI-B3-441-2016. 